\title{
Study on the Method of Speed Detection and Image Recognition Based on Signal Normalized Cut Algorithm
}

\author{
Chunmei Lu \\ Department of Physical Education, Leshan Vocational \& Technical College, Leshan, 614000, \\ China \\ chunmei_lu@yeah.net
}

Keywords: Signal recognition, Normalization, Time series, Image segmentation, Arm speed.

\begin{abstract}
In the image segmentation process, this paper uses the time series similarity principle to divide the image of track and field athletes into many similar blocks, and uses normalized processing to detect and identify the image, which greatly improves the speed and precision of image recognition. In order to verify the effectiveness and the reliability of the algorithm, this paper detects the arm speed of sprinter, finally the image segmentation normalized results and the curve of arm speed changing with time are obtained, which provides a new computer algorithm for identification and detection of arm speed signal.
\end{abstract}

\section{Introduction}

Research on arm speed of track and field athletes has assist in analysis of technical movement, but the image recognition process of arm speed will be affected by many factors, especially they are easily influenced by the scene environment, light and other factors, so it has higher requirements on the image processing $[1,2]$. This paper uses the image segmentation normalized form to process the image, and the specific process is as shown in Figure 1.

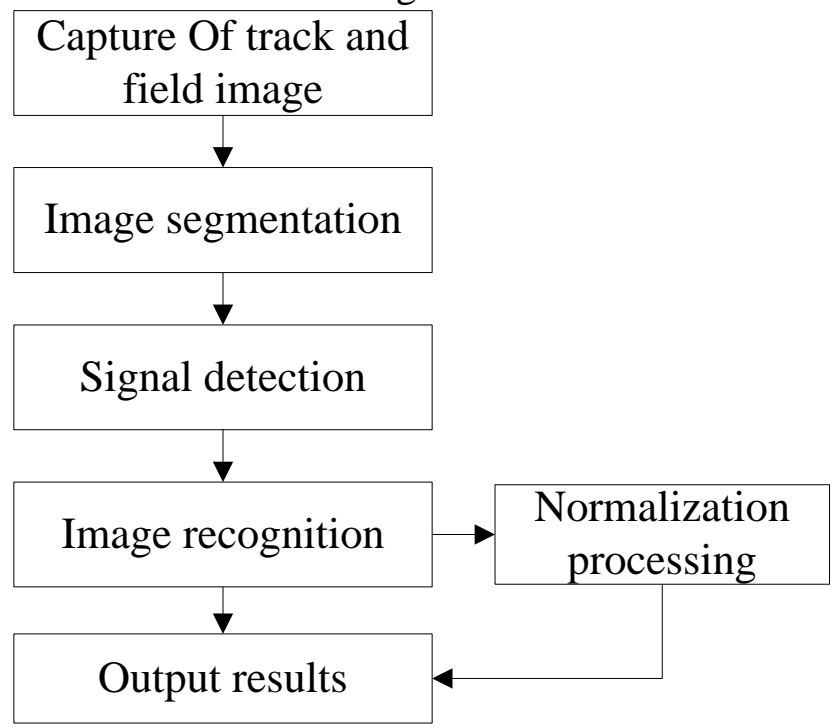

Fig.1: Image segmentation, normalization process

As shown in Figure 1, it indicates design process of image segmentation normalization. From the chart, it can be seen that image segmentation and normalized is mainly based on the principle of image segmentation [3]. Based on the similarity of actions the image can be segmented into many small pieces, then the pieces are done signal normalized processing, finally the arm speed detection and recognition is obtained, which makes image positioning more accurate and rapid.

SCM is the controlling core of this intelligent temperature and humidity detection system. In addition to the temperature and humidity sensor which is served as measuring element, some key techniques, such as temperature and humidity measurement, communication technology and error correction are also used [4]. This system is divided into five parts: temperature measurement circuit, humidity measurement circuit, A/D conversion and filter circuit, data storage and display circuit, 
and the voice alarm circuit. The main devices that selected are: temperature sensor DS18B20, humidity sensor HSll01, AT89S51, A/D converter TLC0834, data memory AT24C04, LCD display module, voice alarm chip ISD2560, MAX232, integrated timer 555 chips and so on.

\section{The Design of Image Segmentation Normalized Recognition Algorithm}

In order to identify the swing arm speed of sprinters, it needs to segment the movement image in a continuous time, finally through the normalization statistics, so as to identify the arm swing speed [5,6]. First assume a continuous time series: $T=t_{1}, t_{2}, \cdots, t_{n}$, and $T$ satisfies the following conditions:

$$
T=\left\{\begin{array}{cc}
y_{1}\left(x, v_{1}\right)+f_{1}(x) & 1 \leq x<b \\
\cdots & b_{k-1} \leq x<b_{k}=M+1 \\
y_{1}\left(x, v_{n}\right)+f_{k}(x) &
\end{array} .\right.
$$

Where $y$ is the coefficient vector, $M$ is ready vector. Its form includes Fu Liye change, and the equation can be expressed as:

$$
\mathrm{T}_{\mathrm{c}}=\sum_{i=1}^{k} T_{i}=\sum_{i=1}^{k} \sum_{j=1}^{s}\left(a b_{j-1}+j-y_{i}\left(b_{i-1}+j, v_{i}\right)\right)^{2} .
$$

Where $a$ is the sampling number in section $i$, according to the time sequence it can segment image. The segmentation effect evaluation index function can be expressed as shown in formula (3).

$$
\mathrm{P}= \begin{cases}\frac{\mathrm{T}}{\sum_{\mathrm{i}=1}^{\mathrm{n}}\left(y_{i}-\bar{y}\right)}+\frac{t}{n} & \sum_{\mathrm{i}=1}^{\mathrm{n}}\left(y_{i}-\bar{y}\right)^{2}>0 \\ \frac{t}{n} & \sum_{\mathrm{i}=1}^{\mathrm{n}}\left(y_{i}-\bar{y}\right)^{2}=0\end{cases}
$$

When $\sum_{i=1}^{\mathrm{n}}(y i-\bar{y})^{2}=0$, time series is a horizontal line.

When $\sum_{\mathrm{i}=1}^{\mathrm{n}}\left(y_{i}-\bar{y}\right)^{2}>0$

$$
p_{j}=g\left(t, v_{j}\right) \quad j=1,2, \cdots, k ; \quad b_{k-1} \leq t<b_{k} .
$$

So, the segmentation normalized formula of image can be obtained.

$$
J=\frac{\sum_{j=1}^{k}<f j, f j>}{\sum_{j=1}^{k}\left(<f j, f j>+\left(<\hat{q}_{j}-1 \bar{y}, \hat{q}_{j}-1 \bar{y}>\right)\right.}+\frac{p}{n} .
$$

Modular design is used in this system. System software includes many subprograms, mainly: the main program and key processing, interruption, actuator control, display and communication. The main program completes device initialization, and determines whether there is key pressed or interruption request. And then according to the judgment result, this system will call for the corresponding subprogram. Figure 2 is program flow diagram. 


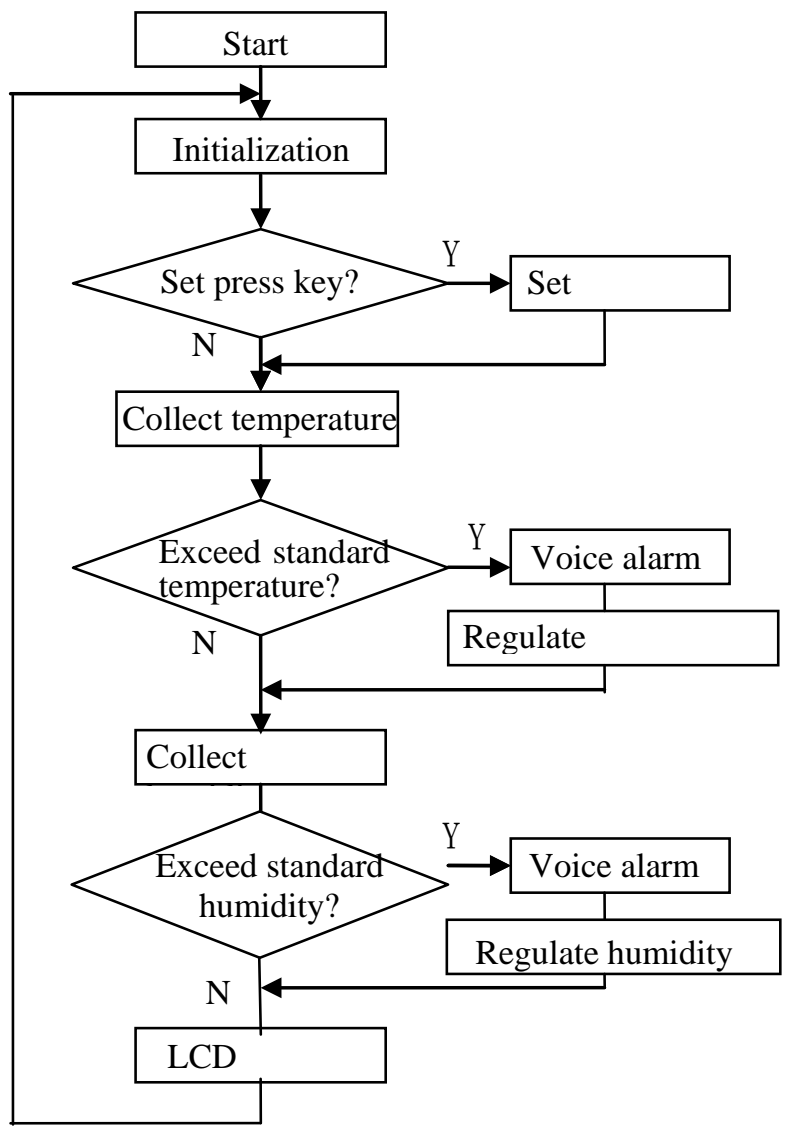

Fig.2: Main program flow diagram

In order to realize the image segmentation normalized, this paper uses MATLAB software to program the algorithm, and the program is as follows:

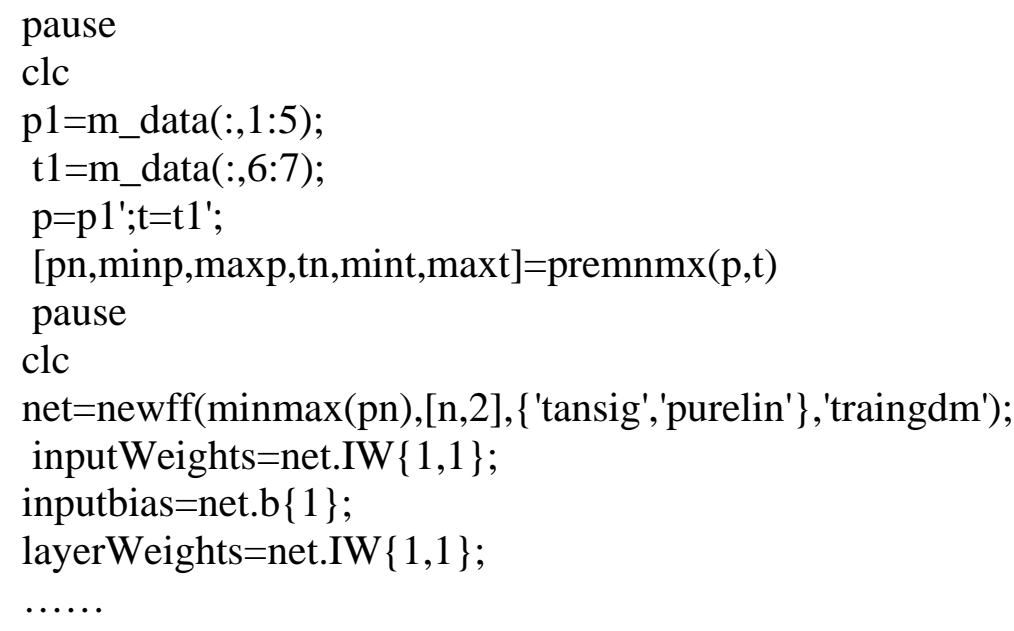

\section{Research of Arm Speed Signal Normalization Recognition for Sprinters}

Based on the image segmentation normalization, firstly the similar motion images are segmented, and the segmentation regional block is as the normalized node, then using the block color histogram to calculate as shown in Figure 3. 


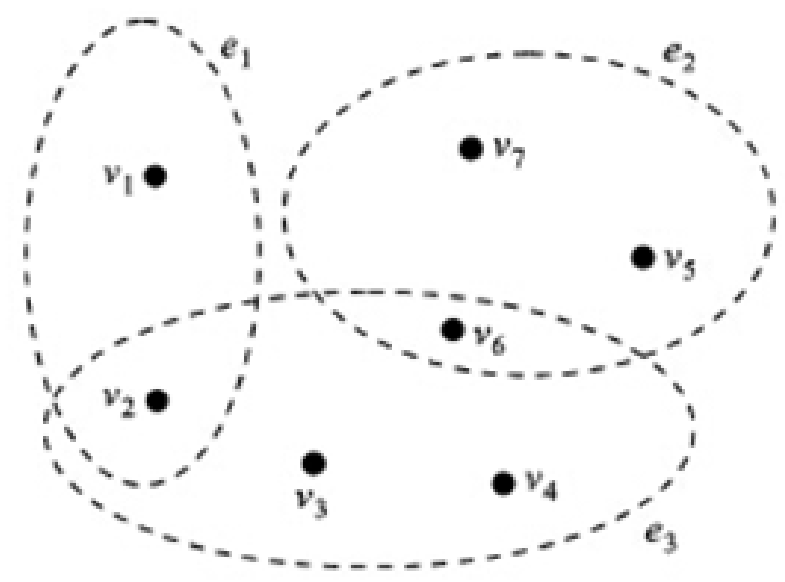

Fig.3: Schematic diagram of image segmentation normalization process

Figure 3 shows the segmentation normalization process of sprint image [7, 8]. Firstly determine similarity of all the inter block, and the corresponding similar node set and a single image adjacent node collection is as the segmentation edge, and calculating the weights, constructing normalized graph as shown in Table 1.

Table 1: Image normalization segmentation

\begin{tabular}{llll}
\hline & b1 & b2 & b3 \\
\hline a1 & 1 & 0 & 0 \\
a2 & 1 & 0 & 1 \\
a3 & 0 & 0 & 1 \\
a4 & 0 & 0 & 0 \\
a5 & 0 & 1 & 1 \\
a6 & 0 & 1 & 1 \\
a7 & 0 & 1 & 0 \\
\hline
\end{tabular}

Table 1 shows the node number of image segmentation normalization. It uses spectrum analysis approximation algorithm to solve the normalization segmentation problem of sprinter action image, finally the results of image segmentation are obtained.

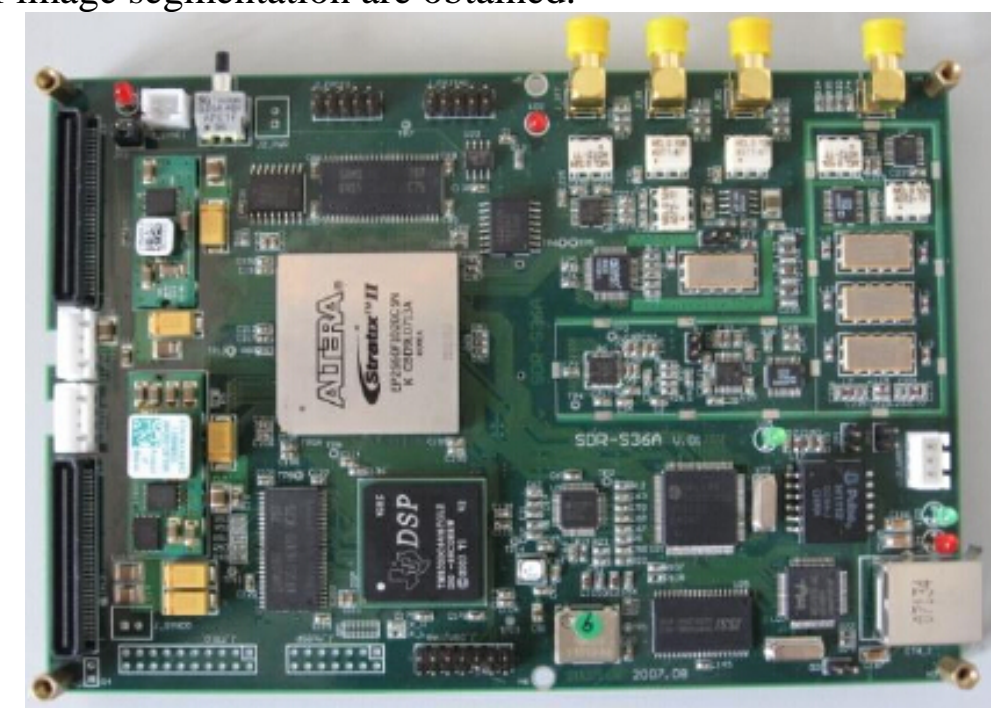

Fig.4: Digital IF processing platform

Figure 4 is the real picture of image segmentation digital IF processing platform [9]. At present, the synthetic systems have been tested and used in multiple application scenarios, and the performance reaches the design target, running in good condition and subjecting to certain users. 


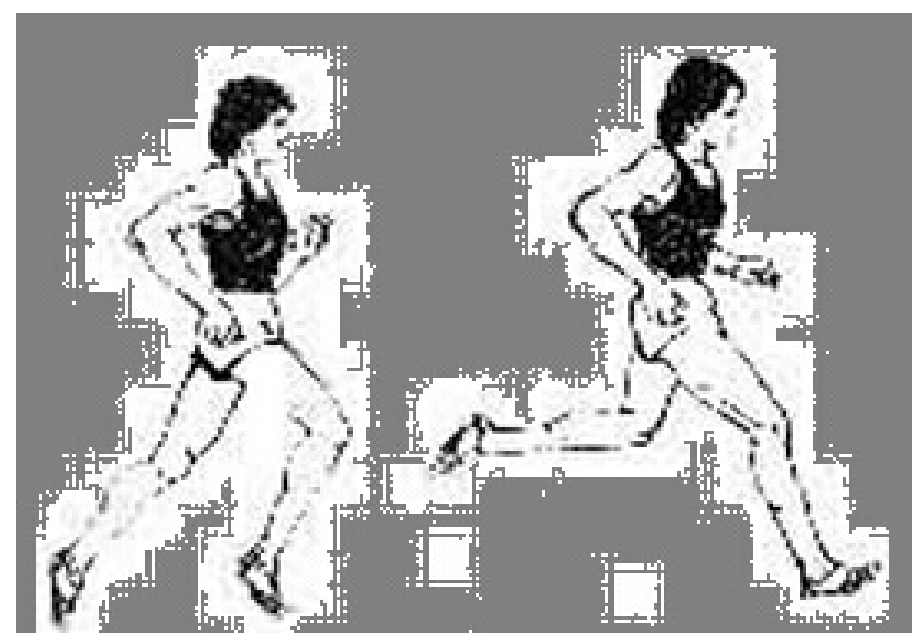

Fig.5: The image segmentation normalization results

Image normalization algorithm is to merge the two adjacent blocks into one block in the same image,as Figure 5 shown. Adjacent is the pixel neighboring in two blocks, so the images are constructed independently normalized image, which can identify the continuous action of images.

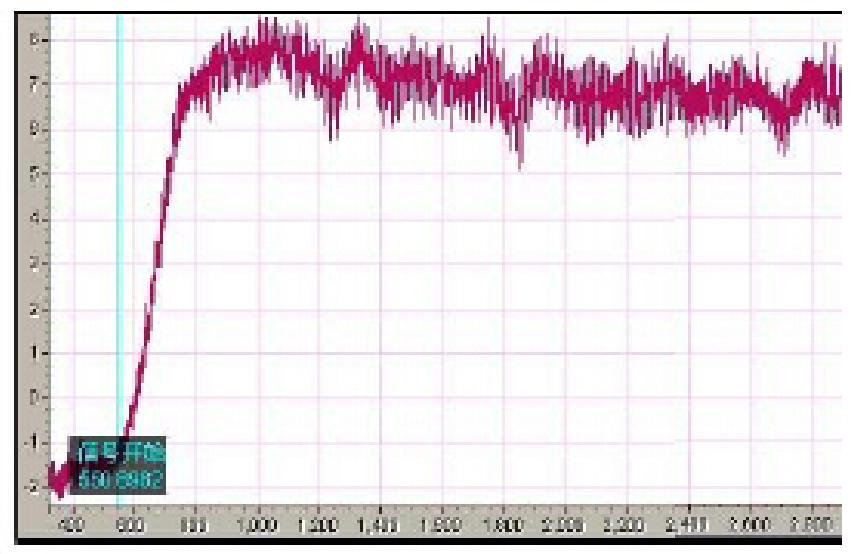

Fig.6: Swing arm frequency distribution in different time

Figure 6 shows the image treatment using normalization segmentation algorithms in continuous time, and swing arm number in different time is obtained. From the chart it can be seen, swing arm number at different moments is different [10]. From the beginning start to speed up the process, the swing arm speed is fast, finally gradually stable, which is consisting with the actual measurement situation.

\section{Summary}

This paper uses time series equivalent segmentation principle to design image segmentation algorithm, and uses the normalization method to detect and recognize signal, finally the normalization mathematical model of arm speed signal detection is obtained, finally uses MATLAB software to program the algorithm. The track and field athletes sprint image is segmented, similar block image segmentation result is obtained. Through the analysis of arm speed curve, the algorithm is consisting with the actual measurement results, which provides a theoretical basis for the identification and detection of arm speed signal.

\section{References}

[1] Y.Z.Jiang, Z.F. Hao. Automatic threshold image segmentation based on block sampling and genetic algorithm. Journal of Computer Aided Design \& computer graphics, 2012, 23(11): 1861-1867.

[2] L.H. Jiang, Z.H. Gan. Review of multi target tracking application. Computer system, 2012, 19(12): 271-273. 
[3] C. Li, X.Y. Cheng. High speed DSP system signal integrity analysis simulation based on Hyperlynx. Electronic devices, 2012, 4(5): 85-89.

[4] P. Tong, Y.H. Hu. FPGA device selection research. Software technology, 2012, 5(2): 45-48.

[5] Y.F. Pu, W. Zhang, S.H. Teng, H.L. Du. A cooperative network intrusion detection based on decision tre. Journal of Jiangxi Normal University: Natural Science Edition, 2013, 34(3): 302307.

[6] Z.J. Zhu, Z.C. Le, R. Zhu. Research and implementation of OBS network simulation platform based on NS2. Journal of communication, 2013, 30(9): 128-134.

[7] L. Zhang, Y. Wang. CAI courseware design based on construction design. Chinese audio-visual education, 2012, 16(2): 41-45.

[8] F.M. Lei. The computer software development in the field of sports. Journal of Xi'an Physical Education University, 2013, 20(2): 113-114.

[9] J. Zhou, R.L. Wang, Y.D. Wu. Classification linear extraction based on edge detection. Journal of Institute of Surveying and mapping, 2014, 25(3): 181-183.

[10] X.L. Zhang. Overview of image edge detection technology. Electronic application technology, 2012, 4(5): 38-40. 